\title{
MAGNETIC BEHAVIOR OF VERY SMALL SUPERCONDUCTING PARTICLES
}

\author{
P. G. DE GENNES and M. TINKHAM* \\ Service de Physique des Solides, Faculté des Sciences, Orsay (Seine et Oise), France
}

(Received 20 June 1964)

\begin{abstract}
This paper discusses the critical field of superconducting particles, or films, much smaller in size than the coherence length and the penetration depth; it is restricted to situations where the order parameter may be taken as constant in space, and where the superconducting transition in the presence of the field is of second order. The critical field calculation is then reduced to a study of the magnetic flux $\Phi$ enclosed by all oneelectron trajectories in the normal state during a prescribed time interval $\mathrm{t}$. We show that (1) if $\Phi$ does not have a completely ergodic behavior at large times, the equation of state is of the BCS type, but with a renormalized, field dependent coupling constant $\mathrm{N}(0) \mathrm{V} \eta(\mathrm{H})$.

(2) if $\Phi$ has a certain ergodic property, the effect of the field is comparable to the effect of paramagnetic impurities, as first pointed out in a particular example by Maki. Among other things there is a region of gapless superconductivity in the (HT) plane.

$A$ thin film in a parallel field with diffuse boundary scattering but no volume defects belongs to case (1). This surprising result is due to a geometrical cancellation of successive contributions to $\Phi$. However, a rather small amount of scattering in the bulk is enough to restore case (2). Numerical values of the resulting critical field are discussed in detail for various ratios of the bulk mean free path 1 to the film thickness d. In the situations of major physical interest the theoretical values are proportional to $\mathrm{d}^{-3 / 2}$ and are in rather good agreement with the experimental data.
\end{abstract}

\section{Introduction}

IT is well-known that the critical field $H_{\mathrm{c}}$ of a thin $(d \ll \lambda)$ superconducting film parallel to the magnetic field is higher than the bulk value $H_{c b}$ by a factor of order $\left(\lambda_{e f f} / d\right)$, where $\lambda_{e f f}$ is the effective penetration depth describing the field penetration and $d$ is the film thickness. This result follows simply from the reduced diamagnetic energy density when the film is thin enough to allow substantial penetration of the field. For example, if $d \ll \lambda_{e f f}$, the simple London theory [1] leads to a first-order transition at

$$
\frac{H_{\mathrm{c}}(T)}{H_{\mathrm{cb}}(T)}=\sqrt{12} \frac{\lambda_{\text {eff }}(T)}{d} \text {. }
$$

Now, this theory is known to be inadequate, since it takes account neither of the non-linearity nor of the non-locality of the electrodynamics of a superconductor. Using the Ginsburg-Landau $[2,3](G-L)$ theory to handle the non-linearity, one finds a second-order transition at

$$
\frac{H_{\mathrm{c}}(T)}{H_{\mathrm{cb}}(T)}=\sqrt{24} \frac{\lambda_{\text {eff }}(T)}{d}(d<\lambda)
$$

\footnotetext{
* Permanent address: Department of Physics, University of California, Berkeley, California, U.S.A.
} 
greater by simply a factor of $\sqrt{2}$.

To give the requisite careful discussion of the region of validity of this result, we must distinguish two characteristic lengths in the superconductor. First, because the G-L theory is local in its electrodynamics, the result will be valid only if the coherence length of Pippard [4] for response to an electromagnetic field, which we denote $\xi_{A}$, is short on the scale of variation of $\mathbf{A}$. For thin films, this scale is set by $d$, since $d \ll \lambda$. Second, the result is derived under the assumption that the film is thin enough so that the gap parameter is uniform over the sample thickness, i.e., that no vortex structure is set up as in a bulk type II superconductor, in which case the critical field would be $H_{\mathrm{c} 2}$ [5].

This requires that $d<\xi_{\Delta}(T)$, where $\xi_{\Delta}(T)$ is the characteristic length for variation of the gap parameter. In view of these two conditions, we require

$$
\xi_{A}<d<\xi_{\Delta}(T)
$$

Now, $\xi_{A}$ is given by Pippard's relation [4]

$$
1 / \xi_{A}=1 / \xi_{0}+1 / 1
$$

where $\xi_{0}=0.18 \hbar v_{F} / k T_{0}$ and 1 is the electronic mean free path. Evidently, $\xi_{A} \approx 1$, if $1 \ll \xi_{0}$. On the other hand, $\xi_{\Delta}(T)$ is given in the G-L approximation [6] by

$$
\xi_{\Delta}(T) \approx \xi_{0}\left(\frac{T_{0}}{T_{0}-T}\right)^{1 / 2} \quad 1 \gg \xi_{0}
$$

or

$$
\xi_{\Delta}(T) \approx\left(\xi_{0} 1 \frac{T_{0}}{T_{0}-T}\right)^{1 / 2} \quad 1 \ll \xi_{0}
$$

where $T_{0}$ is the critical temperature when $H=0$. In a clean film $\left(1>\xi_{0}\right)$, the first part of the inequality (I.3) can only be satisfied very near $T_{0}$ since $\xi_{0}>\lambda(0)$ (in non-transition metals) and since we require that $\lambda>d$ for (I.2) to hold. If one does operate near $T_{0}$ the second part of the inequality (I.3) is readily satisfied. In a dirty film $\left(1 \ll \xi_{0}\right)($ I. 3$)$ is easier to satisfy since $\xi_{A} \sim 1$ which can be made less than $d$, while $\xi_{\Delta} \approx \sqrt{1 \xi_{0}}$ still may exceed $d$, even for $T$ well below $T_{0}$.

Restricting our attention to the dirty case, the BCS theory [7] leads at $T=0$ to:

$$
\lambda_{\text {eff }}=\lambda_{L}(0)\left(\frac{\xi_{0}}{\xi_{A}}\right)^{1 / 2} \approx \lambda_{L}(0)\left(\frac{\xi_{0}}{l}\right)^{1 / 2}
$$

where $\lambda_{L}$ is the London penetration depth defined in the conventional manner in terms of the properties of the pure normal metal. A corresponding relation holds for $T>0$ apart from a numerical factor which reaches 0.86 at $T_{0}[8]$. Combining (I.6) with (I.2) we find for a dirty film

$$
\frac{H_{c}(0)}{H_{c b}(0)}=\sqrt{24} \frac{\lambda_{L}(0)}{d}\left(\frac{\xi_{0}}{1}\right)^{1 / 2}=4.90 \frac{\lambda_{L}(0)}{d}\left(\frac{\xi_{0}}{1}\right)^{1 / 2} .
$$

For $T>0$ a similar relation holds apart from the numerical factor mentioned above.

If the film is clean, so that $l \gg \xi_{0} \gg \lambda>d$ the nonlocality of the electrodynamics must be taken into amount. The important Fourier components of the field have $k \sim 1 / d$ and, since the BCS kernel $K(k) \approx 1 / \lambda_{\text {eff }}^{2}(k)$ relating $\mathbf{J}(k)$ to $\mathbf{A}(k)$ falls as $1 / k \xi_{0}$ (for $k \xi_{0}>1$ ), one can see that this will lead to $\lambda_{\text {eff }} \approx \lambda_{L}\left(\frac{\xi_{0}}{d}\right)^{1 / 2}$. Carrying this through in detail, in the film thin limit, with diffuse surface scattering, one obtains $[9,10]$ 


$$
\begin{aligned}
\frac{H_{\mathrm{c}}(0)}{H_{\mathrm{cb}}(0)} & =8\left(\frac{2}{3}\right)^{1 / 2} \frac{\lambda_{L}(0)}{d}\left(\frac{\xi_{0}}{d}\right)^{1 / 2} \\
& =6.53 \frac{\lambda_{L}(0)}{d}\left(\frac{\xi_{0}}{d}\right)^{1 / 2}
\end{aligned}
$$

which is evidently very similar to (I.7) with $1 \approx d$. Again this relation can be extended to $T>0$ with the same numerical factor as in (1.7).

It should be noted that the results (I.7) and (I.8) have been obtained by assuming that the non-linearity could be treated at least approximately by the G-L theory at low temperature as well as near $T_{0}$, the only region in which it is expected to be really reliable. Experimental results are generally in satisfactory agreement with these simple predictions. In particular, the measured temperature dependence of $H_{\mathrm{c}}(T)$ seems quite close to that predicted by (I.2) using the empirical Gorter - Casimir temperature dependences $H_{c b}(t) \sim\left(1-t^{2}\right)$ and $\lambda_{\text {eff }}(t) \approx\left(1-t^{4}\right)^{-1 / 2}$, where $t=T / T_{c}$. Use of these dependences in (I.2) leads to $H_{\mathrm{c}}(t) \sim\left(1-t^{2} / 1+t^{2}\right)^{1 / 2}$, which certainly gives a semi-quantitative fit to the data. Since the Gorter Casimir dependences are known to be close to those given by the BCS theory [7], it has generally been felt that the theory gave a satisfactory account of the critical field data.

Despite the success of this simple approach via the macroscopic magnetic energy, attempts to provide a more rigorous theoretical treatment of the critical field of thin films at a low temperature - a treatment based directly on the microscopic theory and hence completely independent of the G-L theory - have encountered many difficulties. For example, the variational calculation of Bardeen [11] leads to a first order phase transition at low temperatures, contrary to experimental evidence $[12,13]$ A completely different approach is taken by Nambu and Tuan [14], who pair one-electron states as modified by the field. They correctly obtain a second-order phase transition at all temperatures, but their critical field diverges as

$\sqrt{\ln \left(T_{0} / T\right)}$ as $T \longrightarrow 0$, contrary to experiment, which shows $H_{\mathrm{c}}(T)$ approaching a constant as $T \longrightarrow 0$. A third approach by di Castro and Valatin [15] is somewhat similar in results to that of Nambu and Tuan, except that the critical field at $T=0$ is finite, but typically of order $10^{5}-10^{6}$ gauss and varying as $d^{-5 / 2}$, neither of which is in accord with experiment. In ref. [14] and [15] only ideal films are considered (with specular reflection at the boundaries and $l=\infty)$. The opposite limit of a very dirty film $(l \ll d)$ has been considered by Maki [16]. He formally solves the equation for the one electron propagator in the presence of the field and impurities, then eliminates the impurities by a renormalization procedure, and finally expands the resulting self energy in powers of the field. The critical field thus obtained is given by a formula similar to (I.7) and is thus of the right order of magnitude. A remarkable by-product of the calculation is that in a certain part of the $(H, T)$ diagram one expects gapless superconductivity. The only difficulty of this work is that it involves a number of unclear approximations (i.e., the space dependence of the self-energies is neglected).

For these reasons it was felt that a unified treatment, applicable as well to the clean limit of Nambu and Tuan as to the dirty limit of Maki, and to intermediate cases, would be useful. We have achieved this result by a "method of trajectories" described in Section II. The calculation of the critical field is reduced by a simple transformation to a study of the magnetic flux $\Phi$ enclosed by the one-electron trajectories in the normal state during a prescribed time interval $(0, t)$. The statistical properties of $\Phi$ can be obtained even for rather complicated physical situations (in particular for those where both surface and volume scattering are important). In section III we show that the theoretical behavior in the superconducting state may differ widely, depending on whether $\Phi$ has, or has not, a certain ergodic property at large times. The situation considered by Nambu and Tuan is non-ergodic, while the "dirty superconductor" case of Maki is ergodic. The central problem is then to find the behavior of physically more important intermediate cases, such as a thin film in a parallel field with diffuse scattering but no defects in the bulk. It is shown in section IV that for such a film $\Phi$ is not ergodic. (The film does not behave like a Maki dirty superconductor with an effective mean free path comparable to the thickness.) This surprising result is due to a geometrical cancellation of successive contributions to $\Phi$. However, we also show that a rather small amount of impurity scattering in the bulk is enough to restore the ergodic properties. When this condition is realized, we in fact obtain a critical field very similar in form to (I.8), which is known to be a good approximation to the experimental facts. 


\section{The Method of Trajectories}

Our starting point is the observation that, in small samples, the normal superconducting transition is of second order, even in the presence of the magnetic field, as shown by a number of experiments $[12,13]$. Then, at the critical temperature, the pair potential $\Delta(\mathbf{r})=V\left\langle\psi_{\uparrow}(\mathbf{r}) \psi_{\downarrow}(\mathbf{r})\right\rangle$ is ruled by a linear selfconsistency equation [26]

$$
\Delta\left(\mathbf{r}_{1}\right)=V T \sum_{\omega} \mathscr{G}_{\omega}\left(\mathbf{r}_{1} \mathbf{r}_{2}\right) \mathscr{G}_{-\omega}\left(\mathbf{r}_{1} \mathbf{r}_{2}\right) \Delta\left(\mathbf{r}_{2}\right) d \mathbf{r}
$$

where $V$ is the BCS interaction parameter, $\mathscr{G}_{\omega}$ is a thermal Green's function, $\hbar \omega=(2 \nu+1) \pi k_{B} T$, and the sum $\sum_{\omega}$ is extended over all algebraic integers $\nu . \mathscr{G}_{\omega}$ can be written down explicitly in terms of the oneelectron wave functions $\phi_{n}$ in the normal metal defined by

$$
\left[\frac{1}{2 m}\left(p-\frac{e A}{c}\right)^{2}+U(\mathbf{r})-E_{F}\right] \phi_{n}(\mathbf{r})=\xi_{n} \phi_{n}(\mathbf{r}) .
$$

$(U(\mathbf{r})$ is the one-electron potential including the effects of impurities, boundaries, etc.....) We have

$$
\mathscr{G}_{\omega}\left(\mathbf{r}_{1} \mathbf{r}_{2}\right)=\sum_{n} \frac{1}{i \omega \hbar-\xi_{n}} \phi_{n}^{*}\left(\mathbf{r}_{2}\right) \phi_{n}\left(\mathbf{r}_{1}\right)
$$

The highest temperature $T$ at which equation (II.1) has a non-zero solution $\Delta(\mathbf{r})$ is the transition temperature $T_{c}(H)$. To discuss equation (II. 1$)$ we shall first transform its kernel and write it in terms of one-electron correlation functions in the normal state.

a) In zero field this transformation is very easily achieved [18]. We have explicitly

$$
\mathscr{G}_{\omega}\left(\mathbf{r}_{1} \mathbf{r}_{2}\right) \mathscr{G}_{-\omega}\left(\mathbf{r}_{1} \mathbf{r}_{2}\right)=2 \pi N(0) \int_{0}^{\infty} d t \mathrm{e}^{-2|\omega| t}\left\langle\delta\left(\mathbf{r}(0)-\mathbf{r}_{1}\right) \delta\left(\mathbf{r}(t)-\mathbf{r}_{2}\right)>.\right.
$$

$$
(\mathbf{A}=0)
$$

The average in equation (II.4) is taken over all one-electron states at the Fermi level in the normal metal. $\mathbf{r}(t)$ is the time dependent coordinate of the electron when it is submitted to the forces described by $U(\mathbf{r})$. Equation (II.4) can be verified by taking matrix elements between states $\phi_{n}, \phi_{m}$ and using the fact that for
$\mathbf{A}=0$ the $\phi$ 's can be chosen as real [18].

b) In a non-zero field $\mathrm{H}$ the generalization of equation (II.4) is simply:

$$
\mathscr{G}_{\omega}\left(\mathbf{r}_{1} \mathbf{r}_{2}\right) \mathscr{G}_{-\omega}\left(\mathbf{r}_{1} \mathbf{r}_{2}\right)=2 \pi N(0) v \int_{0}^{\infty} d t e^{-2|\omega| t} f\left(\mathbf{r}_{1} \mathbf{r}_{2} t\right)
$$

$v$ is the sample volume and

$$
f\left(\mathbf{r}_{1} \mathbf{r}_{2} t\right)=\left\langle\left[\exp \frac{2 \pi i}{\Phi_{0}} \int_{\mathbf{r}_{1}}^{\mathbf{r}_{2}} \mathbf{A} \cdot \mathbf{d} l\right] \ldots \delta\left(\mathbf{r}(0)-\mathbf{r}_{1}\right) \delta\left(\mathbf{r}(t)-\mathbf{r}_{2}\right)\right\rangle
$$

The integral $\phi=\int 2 \pi \frac{\mathbf{A} \cdot \mathbf{d} l}{\Phi_{0}}$ in equation (II.5) is taken along the classical trajectory linking points $\mathbf{r}_{1}$ and 
$\mathbf{r}_{2}$ in a time interval $t . \Phi_{0}$ is the flux quantum. Equation (II.5) applies only when the cyclotron radius is very large compared with all distances of interest. The proof of (II.5) proceeds as follows:

We first transform the $\mathscr{G}_{\omega}$ 's in terms of real time Green's functions $\hat{\mathscr{G}}$ defined by

$$
\begin{gathered}
\hat{\mathcal{G}}\left(\mathbf{r}_{1} \mathbf{r}_{2} t\right)=\sum_{n} \phi_{n}^{*}\left(\mathbf{r}_{2}\right) \phi_{n}\left(\mathbf{r}_{1}\right) e^{-i\left(E_{F}+\xi_{n}\right) t / \hbar} \\
\mathscr{G}_{\omega}\left(\mathbf{r}_{1} \mathbf{r}_{2}\right)=-i \sigma \int_{0}^{\infty} d t \mathrm{e}^{i \sigma_{E_{F} t / \hbar}} e^{-|\omega| t} \mathscr{G}(\sigma t) \\
\sigma=\frac{\omega}{|\omega|} \\
\mathscr{G}_{\omega}\left(\mathbf{r}_{1} \mathbf{r}_{2}\right) \mathscr{G}_{-\omega}\left(\mathbf{r}_{1} \mathbf{r}_{2}\right)=\int_{0}^{\infty} d t \int_{0}^{\infty} d t^{\prime} \mathrm{e}^{i E_{F}\left(t-t^{\prime}\right) / \hbar} e^{-|\omega|\left(t+t^{\prime}\right) \hat{\mathcal{F}}\left(\mathbf{r}_{1} \mathbf{r}_{2} t\right) \hat{\mathscr{G}}\left(\mathbf{r}_{1} \mathbf{r}_{2} t^{\prime}\right)}
\end{gathered}
$$

We now observe that the times of interest are of order $\omega^{-1} \sim \hbar / k_{B} T$ and that the corresponding spatial distances are very large compared with the Fermi wavelength. Then we can go to the classical limit, where

$$
\mathscr{G}\left(\mathbf{r}_{1} \mathbf{r}_{2} t\right)=W\left(\mathbf{r}_{1} \mathbf{r}_{2}\right) e^{i S(0 t) / \hbar}
$$

where $S(0 t)=\int_{0}^{t} d r\left(\frac{1}{2} m v^{2}+\frac{e A}{c} \cdot v-U(r)\right)$ is the action calculated along the classical trajectory relating $\mathbf{r}_{1}$ to $\mathbf{r}_{2}$ in time $t$ (the instantaneous velocity being $v$ ). $W$ is an amplitude factor and is independent of $A$ in the limit of very large cyclotron radius. We insert (II.9) in (II.8) and notice that, because of the factor $e^{i E_{F}\left(t-t^{\prime}\right) / \hbar}$ the only non-zero contributions to (II.8) will come from $t^{\prime}$ nearly equal to $t$. Then the total phase of the product $\hat{\mathscr{G}} \hat{\mathscr{G}}$ is simply $\hbar^{-1}[S(0 t)+S(0,-t)]$. If the curvature of the trajectories due to the field is negligible, the two actions are computed on the same path; the terms $\frac{1}{2} m v^{2}-U$ cancel out and we are left with a phase $\phi$ given by an integral along the classical trajectory:

$$
\phi=\int_{0}^{t} \frac{2 \mathrm{eA}}{h \mathrm{e}} \cdot \mathbf{v} d t=\frac{2 \pi}{\Phi_{0}} \int \mathbf{A} \cdot \mathrm{d} 1 .
$$

Apart from the factor $e^{i \phi}$ equation (II.8) has the same structure as in the case $\mathbf{A}=0$ and referring to (II.4) we obtain (II.5). A phase factor equivalent to (II.10) has been derived by Gor'kov [19] for electrons in an infinite pure metal, where the trajectories are straight lines. The main interest of the present derivation is to cover cases where impurity and boundary scattering may be important.

Having now constructed the kernel of our initial equation (II.1) we further restrict our attention to physical situations where $\Delta(r)$ has a constant amplitude over the whole volume of the sample. This excludes all configurations of the vortex type, and is probably correct for particles of dimensions $d \ll \xi \Delta$ and for thin films with a field strictly parallel to the film plane. Then it is convenient to choose for the vector potential $\mathbf{A}$ that particular gauge where $\Delta$ is real (and constant) in the whole specimen. This enables us to integrate equation (II.1) over $r_{1}$. Dividing both sides by $v \Delta$ and inserting (II.5) for the kernel we obtain the condition

$$
1=2 \pi T N(0) V \sum_{\omega} \int_{0}^{\infty} d t e^{-2|\omega| t}\left\langle e^{i \phi(t)}\right\rangle
$$


The phase $\phi$ is defined by equation (II.10) and the average in (II.11) is taken over all initial positions and over all initial orientations of the velocity (of length $v_{F}$ ) for one electron in the sample. Equation (II.11) reduces the $T_{c}(H)$ calculation to a study of all classical one-electron trajectories in the normal state. The main interest of the present method is to show that the (apparently complicated) behavior in the superconducting phase is in fact controlled by some simple geometrical properties of the trajectories.

Equation (II.11) is a particular case of a more general formula giving the transition temperature in the presence of both orbital magnetic fields and magnetic impurities, namely

$$
1=2 \pi T N(0) V \sum_{\omega} \int_{0}^{\infty} d t \mathrm{e}^{-2|\omega| t}\left\langle K^{+}(0) K(t)\right\rangle
$$

Here $K$ is the time reversal operator (acting on the one-electron states $\phi_{n}$ ) and

$$
K(t)=e^{i H_{0} t / \hbar} K e^{-i H_{0} t / \hbar}
$$

is the corresponding Heisenberg operator. A formula equivalent to (II.12) is derived in ref. [17] (equation II.2). Equation (II.12) applies when (a) the transition is of second order, (b) the Cooper pairs are in s-states, (c) $|\Delta|$ is constant in space. For non-magnetic impurities, in zero field, $K$ commutes $\mathscr{H}_{0}$, $K^{+} K(t)=K K^{+}=1$ and (II.12) reduces to the BCS condition (Anderson's Theorem). For magnetic impurities $K$ acquires a time dependent phase

$$
\phi_{\text {mag }}=2 \int H_{\text {ex }}(t) d t
$$

where $H_{e x}$ is the exchange field (in frequency units), due to the impurities, acting on the electron spins. Here, with orbital fields, the phase of $K$ is given by equation (II.19). This unified formulation gives a somewhat deeper understanding of some analogies between magnetic impurity effects and magnetic field effects which we shall encounter later on.

\section{The Two Types of Magnetic Behavior}

As already pointed out, we are interested mainly in the limiting behavior of the average $\left\langle\mathrm{e}^{i \phi(t)}\right\rangle$ at large times $t$. It turns out that sometimes $\left\langle\mathrm{e}^{i \phi}\right\rangle$ has a non-zero limit $\eta$ for $t \longrightarrow \infty$, while in many other situations it decays exponentially towards zero.

$$
\lim _{t \rightarrow \infty}<e^{i \phi(t)}>= \begin{cases}\eta \neq 0 & \text { case I } \\ e^{-t / \tau_{K}} & \text { case II }\end{cases}
$$

(where $\eta$ and $\tau_{K}$ are functions of the applied field).

Of course (I) and (II) do not exhaust all possibilities but they cover all the various examples to be discussed below. The magnetic properties of a given sample will be very different depending on which case is
obeyed.

Case I: we may write

$$
\left\langle\mathrm{e}^{i \phi(t)}\right\rangle=\eta+R(t)
$$

where $R(t)$ decays towards zero for time $t \tilde{>} d / v_{F}$. Since $d / v_{F} \ll \hbar / k_{B} T$ we may neglect $R(t)$ completely at the times of interest. Then (II.2) take the usual BCS form [7]

$$
1=N(0) V \eta(H) \int_{-\hbar \omega_{D}}^{\hbar \omega_{D}} \frac{1-2 f(\xi)}{2 \xi} d \xi
$$


so that:

$$
k_{B} T=1.14 \hbar \omega_{D} \exp \left(-\frac{1}{N(0) V \eta(H)}\right) .
$$

Thus in case I the behavior is close to that of a conventional superconductor except for the fact that the coupling constant $N(0) V \eta$ is a (decreasing) function of the applied field $H$. The transition temperature $T$ remains finite at all fields, but in fact becomes exponentially small as soon as $\eta$ goes below $\sim 1 / 2$. A renormalization factor of this sort has been encountered by Nambu and Tuan [14] for a thin film with specular reflexion at the boundaries - a typical example of case I. We shall see later that even with diffuse scattering on the boundaries, in an otherwise ideal film, one still obtains case I.

Case II: If the exponential decay law (III.2) is already obeyed at the times $t$ of interest we may write:

$$
1=2 \pi T N(0) V \sum_{\omega} \frac{1}{2|\omega|+1 / \tau_{K}} .
$$

By standard transformations this leads to:

$$
\log \frac{T_{0}}{T_{c}}=\psi(1 / 2)-\psi\left(1 / 2+\frac{\hbar}{4 \pi k_{B} T_{c} \tau_{K}}\right) .
$$

Here $T_{0}$ is the transition temperature in 0 field, $T_{c}$ is the transition temperature with the field, and $\psi(z)=\frac{\Gamma^{\prime}(z)}{\Gamma(z)}$. Equation (III.7) is an implicit equation for $T_{c}$. The field dependence comes through $1 / \tau_{K}$, which we shall see is proportional to $H^{2}$ in most cases. A plot of $\frac{T_{c}}{T_{0}}$ as a function of $1 / \tau_{K}$ is given in

Fig. 1. $T_{c}$ decreases with increasing $1 / \tau_{K}$ and finally vanishes for

$$
1 / \tau_{K}=1.76 \frac{k_{B} T_{0}}{\hbar} .
$$

Equation (III.7) is familiar from the problem of paramagnetic impurities in superconductors [20] where (for very different reasons) the time reversal operator $K$ also has a Lorentzian power spectrum [17]. (In the latter case $\frac{1}{\tau_{K}}$ is equal to $\frac{2}{\tau_{s}}$ where $\tau_{s}$ is the lifetime of a one-electron plane wave state due to the exchange interaction with the impurities.)

Because the implicit nature of (III.7) obscures its significance, we have plotted the result in Fig. 1, namely, the solution of (III.7) for $t_{c}(h)=T_{c}(h) / T_{0}$, where $h=H / H_{c}(0)$ in this case. To make this plot more meaningful, we have also plotted for comparison the function

$$
t_{c}(h)=\left(\frac{1-h^{2}}{1+h^{2}}\right)^{1 / 2}
$$

which arises from the insertion of the temperature dependences of the Gorter-Casimir two fluid model in (I.2) and solving for $t_{c}(h)$ rather than the more usual $h_{c}(t)$. It is apparent that the two functional dependences are quite similar. Since experimental data are known to be well approximated by (III.9), this comparison shows that the temperature dependence of $h_{c}$ given by this theory is at least in reasonable agreement with experiment. We note in particular that $h_{c}(t)$ approaches a constant $h_{c}(0)$ in the present theory, rather than displaying the logarithmic divergence at $T=0$ characteristic of the theory of Nambu and Tuan. Careful measurements of $h_{c}(t)$ on very thin films over a wide range of temperature would be desirable to test the theory more precisely. To the extent that a comparison can be made with recent data of Douglass and Blumberg [21] near $T_{c}$ and of Toxen [22] over a wider range of temperature, it appears that the experimental dependence lies roughly midway between the two curves in Fig. 1. This may be related to the fact 


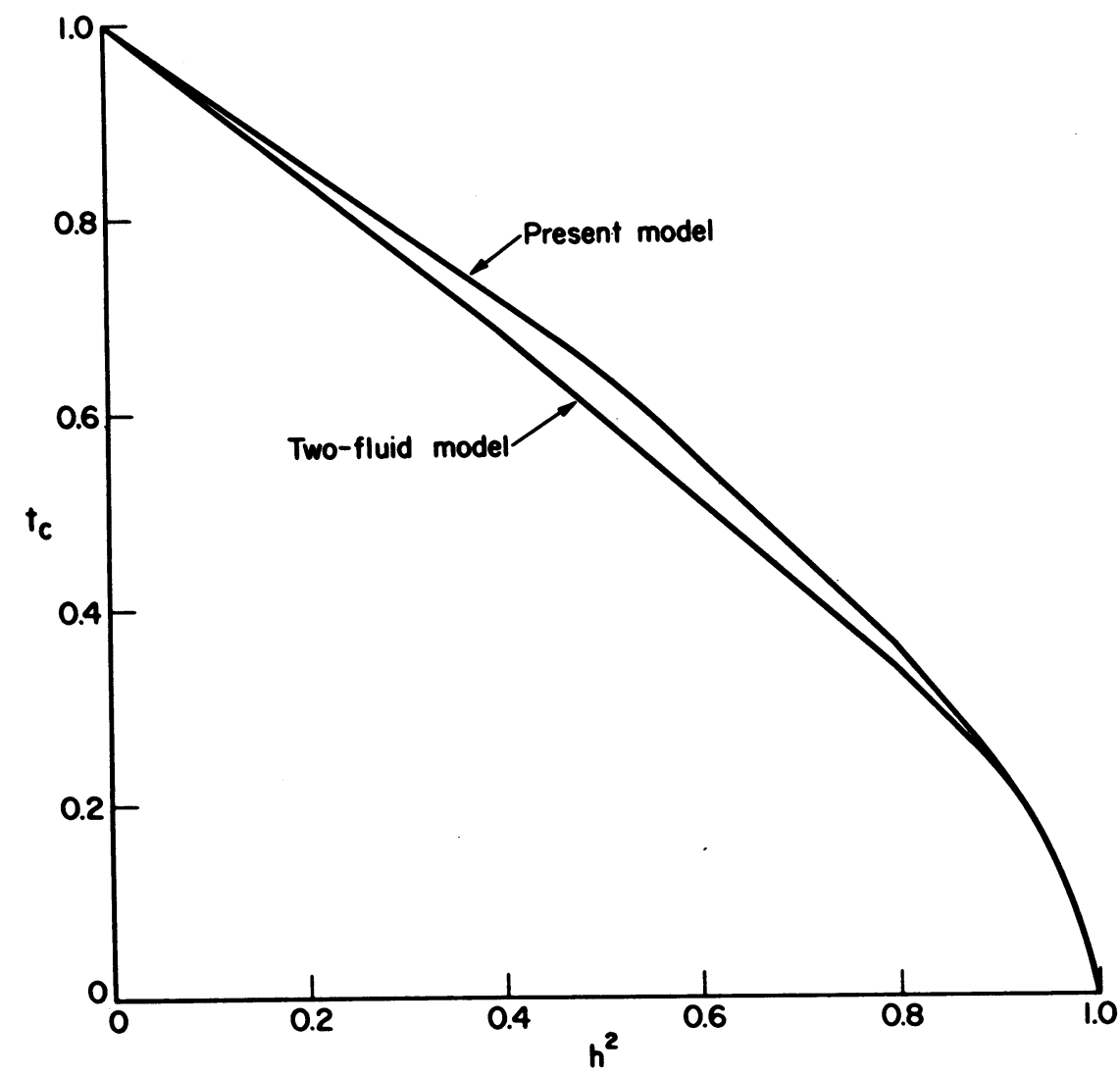

FIGURE 1

Comparison of the field dependences of the critical temperature of a thin film as given by the present model and by the Gorter-Casimir two-fluid model.

$$
\left[t_{c}=T_{c}(H) / T_{c}(0), h=H / H_{c}(0)\right] .
$$

The latter model predicts $h_{c}=\left(1-t^{2} / 1+t^{2}\right)^{1 / 2}$ or, equivalently, $t_{c}=\left(1-h^{2} / 1+h^{2}\right)^{1 / 2}$. Recent experimental data of Douglass and Blumberg and of Toxen seem to lie between the curves in the Figure, to the extent that a comparison has been made. The gapless region mentioned in the text lies just to the left of the curve given by the present model.

that the BCS theory overestimates the departures from a parabolic $\left(1-t^{2}\right)$ dependence of $H_{c b}$ on temperature. Such an error would be expected to carry over into the present calculation.

A remarkable property associated with a non-singular power spectrum for $K$, as is obtained in case II, is the existence of gapless superconductivity in a small but finite region of the $(H, T)$ plane [20]. (For a simple argument showing how gapless superconductivity occurs when case II applies, see ref. [17]). It may be in fact that small samples of superconducting metals or non-magnetic alloys, when they belong to case II, give us a neater example of gapless superconductivity than the magnetic alloys where many parasitic effects related to impurity - impurity interactions complicate the picture [17].

Finally, for the specific examples to be discussed below, it is important to estimate the order of magnitude of the times $t$ which are of interest in an actual calculation of $\left\langle e^{i \phi(t)}\right\rangle$. When case II is obeyed we have $t \sim \tau_{K}$. For a qualitative estimate of $\tau_{K}$ we may write, instead of (II.12):

$$
\frac{\hbar}{\tau_{K}} \approx k_{B}\left(T_{0}-T_{c}\right)
$$

and we thus obtain

$$
t \approx \frac{\hbar}{k_{B}\left(T_{0}-T_{c}\right)}
$$


The conclusion is that the times of importance are at least of order $\frac{\hbar}{k_{B} T_{0}}$, and even much longer when the field is small and thus $T_{c}$ close to $T_{0}$.

\section{Discussion of Specific Examples}

1) - Dirty superconductor limit. In this section we consider a sample of dimensions $d$ much larger than the mean free path 1 . As indicated in the Introduction, it is important to realize however that there is an upper bound on $d$, related to our assumption $|\Delta|=$ const. At larger $d$ 's, we know in fact that the order parameter is not constant in space: the superconducting phase nucleates at a field $H_{c 2}$, the nuclei having a size $\sim \sqrt{\xi_{0} l \frac{T_{0}}{T_{0}-T}}$ where $\xi_{0}=0.18 \frac{\hbar v_{F}}{k_{B} T_{0}}$. Thus, to be sure that $|\Delta|$ is constant, we must have $d<\sqrt{\xi_{0} l \frac{T_{0}}{T_{0}-T}}$ and the inequalities $1<d<\sqrt{\xi_{0} l \frac{T_{0}}{T_{0}-T}}$ allow only for a rather narrow range of $d$ values. We discuss this region, however, since it is a simple example of case II. From the point of view of our trajectories, the condition $\sqrt{\xi_{0} l}>d$ means that the diffusion length

$$
\sqrt{D t}\left(\text { where } D=\frac{1}{3} v_{F} 1 \text { and } t \sim \frac{\hbar}{k_{B}\left(T_{0}-T\right)}\right)
$$

is larger than $d$, and consequently that at time $t$ the electron has explored all regions of the sample. Then the phase $\phi(t)$ is a sum of many uncorrelated increments and has a gaussian distribution

$$
\left\langle\mathrm{e}^{i \phi(t)}\right\rangle=\mathrm{e}^{-1 / 2\left\langle\phi^{2}(t)\right\rangle} .
$$

We write $\phi(t)=\int_{0}^{t} d t^{\prime} \omega\left(t^{\prime}\right)$ with $\omega=\frac{2 e}{\hbar_{c}} \mathbf{v} \cdot \mathbf{A}$. At times $t$ much larger than the collision time $\tau=1 / v_{F}$ we have:

$$
\left\langle\phi^{2}\right\rangle \longrightarrow 2 t \int_{0}^{\infty}\left\langle\omega(0) \omega\left(t^{\prime}\right)\right\rangle d t^{\prime} . \quad(t \gg \tau)
$$

For trajectories originating from point $\mathbf{r}$ we may write

$$
\begin{array}{rlr}
\left\langle\omega(0) \omega\left(t^{\prime}\right)\right\rangle & =\left(\frac{2 e}{h c}\right)^{2} \sum_{\alpha \beta} A_{a}(\mathbf{r}) A_{\beta}\left(\mathbf{r}^{\prime}\right)\left\langle v_{a}(0) v_{\beta}\left(t^{\prime}\right)\right\rangle & \\
& =\left(\frac{2 e}{h c}\right)^{2} A^{2}(r) \frac{v_{F}^{2}}{3} e^{-t^{\prime} / \tau} . & \alpha, \beta=x, y, z
\end{array}
$$

Since at times of order $\tau$ the change of $\mathbf{r}$ along the trajectory is $\approx 1$ and negligible on the scale of variation of $A(\sim d)$. Then

$$
\begin{aligned}
& \left\langle e^{i \phi(t)}\right\rangle=e^{-t / \tau_{K}} \quad(t>\tau) \\
& \frac{1}{\tau_{K}}=\frac{1}{3} \tau \frac{2 e v_{F}}{h c}\left\langle A^{2}(r)\right\rangle .
\end{aligned}
$$

The average in equation (IV.4) is over all points in the sample. A result nearly equivalent to (IV.4) has been obtained by Maki [16] (through an approximate calculation of the one-electron propagators), the main 
difference being that, a) the present derivation gives a somewhat clearer insight on the approximations made, b) in equation (IV.4) $\tau$ is a transport mean free time - while in reference [16] it is the lifetime of an electron state. (That the transport time should in fact be the one involved has been noticed by Maki.)

It is very important to realize that the choice of gauge in (IV.4) is imposed by the condition: $\Delta=$ const.

a) For simple geometries this gauge can be found by inspection

- thin film (of thickness $d$ along $O x$ ) in uniform field (along $O z$ ), carrying no overall current.

$$
\begin{aligned}
A_{x} & =A_{z}=0 \\
A_{y} & =H x \quad \text { (where } \mathrm{x} \text { is measured from the mid-plane of the film) } \\
<A^{2}> & =\frac{H^{2} d^{2}}{12}
\end{aligned}
$$

- spherical grain of radius $R$ in uniform field

$$
\begin{aligned}
\mathbf{A} & =\frac{1}{2} \mathbf{r} \times \mathbf{H} \\
<A^{2}> & =\frac{H^{2} R^{2}}{10} .
\end{aligned}
$$

b) For more irregular sample shapes, the exact form of $\mathbf{A}(\mathbf{r})$ in the gauge of interest cannot be found so easily. For very impure specimens, where the bulk mean free path is short, there is a local, linear relation between the supercurrent $j_{s}(\mathbf{r})$ and the vector potential $\mathbf{A}(\mathbf{r})$. If the coefficient in this relation is independent of $\mathbf{r}$ (homogeneous system), then $\mathbf{A}(\mathbf{r})$ must satisfy the equations

$$
\begin{aligned}
& \operatorname{div} \mathbf{A}=0 \\
& \mathbf{n} \cdot \mathbf{A}=0 \quad \text { on the surface }
\end{aligned}
$$

i.e., A must be chosen in the London gauge. The second condition expresses the assumption that no external currents are passing through the specimen [23].

2) - Thin film with diffuse reflection on boundaries but no volume defects. The remarkable result is that these films belong to case I

$$
\lim _{t \rightarrow \infty}\left\langle K^{+}(0) K(t)>=\eta \neq 0 .\right.
$$

In spite of the numerous collisions which take place, the operator $K$ does not have an ergodic behavior. This can be understood in the following way: consider an electron starting at point $\mathbf{r}_{0}$ at time 0 (Fig. 2), then suffering collisions alternatively on both sides of the film at times $t_{1}, t_{2}, \ldots, t_{n}$ and finally arriving at $\mathbf{r}_{t}$ at time $t$. We may write

$$
\phi=\Delta \phi_{01}+\Delta \phi_{12}+\ldots+\Delta \phi_{n-1 n}+\Delta \phi_{n t} .
$$

We shall now show that each of these increments (except $\Delta \phi_{01}$ and $\Delta \phi_{n t}$ ) is in fact equal to 0

$$
\Delta \phi_{p p+1}=\frac{2 e H}{\hbar c} \int_{x=-d / 2}^{x=d / 2} x d y=0
$$

Since for all fields of interest the cyclotron radius is very large when compared to $d=2 a$, the trajectory between two successive collisions is essentially a straight line; $y$ is a linear function of $x$. Thus the above 


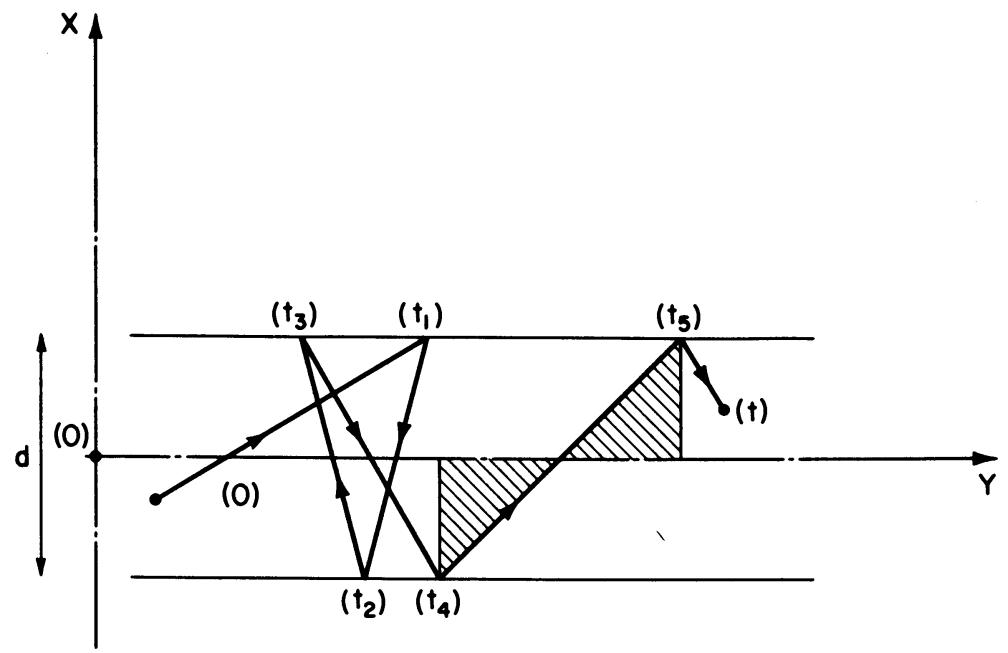

FIGURE 2

A typical trajectory for one electron in a film with diffuse surface scattering and no volume defects (the figure shows the projection of the trajectory on a plane normal to the field direction $z$ ). The increment in phase $\Delta \phi_{45}$ between $t_{4}$ and $t_{5}$ is proportional to the algebraic area

$$
\int_{t_{4}}^{t_{5}} x d y
$$

(hatched area) and vanishes by symmetry. This implies a non-ergodic behavior for $\phi$.

integral, taken between even limits, vanishes and $\phi=\Delta \phi_{01}+\Delta \phi_{n t}$. Furthermore, when $t \gg d / v_{F}$ the number of collisions $n$ is large and the relevant time intervals $0 \longrightarrow t_{1}$ and $t_{n} \longrightarrow t$ are uncorrelated. We may thus write

$$
\eta=\left\langle e^{i \Delta \phi_{01}}\right\rangle\left\langle e^{i \Delta \phi_{n t}}\right\rangle \quad \eta^{1 / 2}=\left\langle e^{i \Delta \phi_{01}}\right\rangle
$$

To compute $\eta$ we consider an electron starting from an arbitrary point $\mathbf{r}$, with a randomly oriented velocity $v_{F}$, the projection of this velocity on the $x y$ plane making an angle $\psi$ with the $x$ axis.

$\eta^{1 / 2}=\frac{1}{\pi d} \int_{-\pi / 2}^{\pi / 2} d \psi \int_{-d / 2}^{d / 2} d x \exp \left[\frac{i \pi H}{\Phi_{0}}\left(d^{2} / 4-x^{2}\right) \tan \psi\right]=\frac{2}{d} \int_{0}^{d / 2} d x \exp \left[\frac{\pi H}{\Phi_{0}}\left(x^{2}-d^{2} / 4\right)\right]$

where $\Phi_{0}=\frac{c h}{2 \mathrm{e}}$ is the flux quantum.

The dependence of $\eta$ on $\frac{H}{\Phi_{0}} d^{2}$ is shown in Fig. 3. The limiting values are

$$
\eta=\begin{array}{ll}
1-\frac{\pi}{3} \frac{H}{\Phi_{0}} d^{2} & H \ll \frac{\Phi_{0}}{d^{2}} \\
{\frac{2 \Phi_{0}}{\pi H d^{2}}}^{2} & H \gg \frac{\Phi_{0}}{d^{2}} .
\end{array}
$$

3) - Thin film with diffuse surface scattering + small amount of defects in the bulk. We shall now see that the presence of a few scattering centers in the bulk (characterized by a mean free path $1>d$ ) brings a 


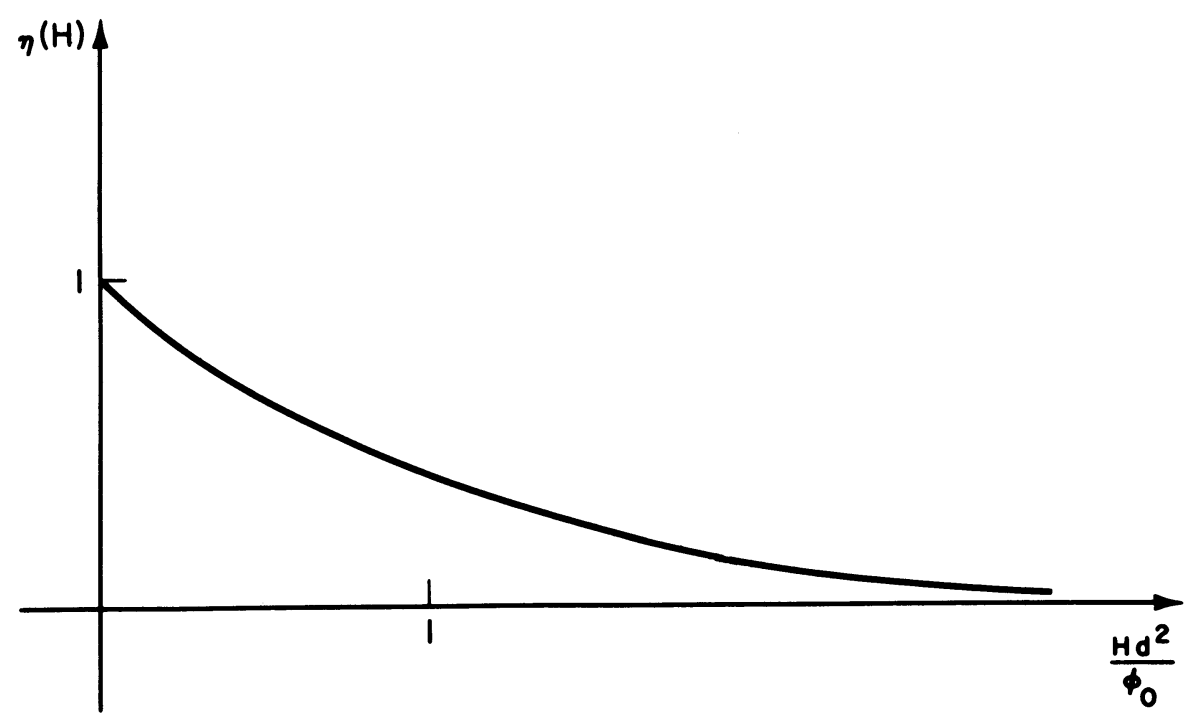

FIGURE 3

Field dependent coupling constant for a thin film with diffuse surface scattering but no volume defects. $\eta(H)$ is the reduced coupling constant.

$$
\frac{(N(0) V)_{\text {eff }}}{N(0) V}=\eta(H)
$$

transition from case I to case II as soon as $1<\xi_{0} \frac{T_{0}}{T_{0}-T}$

a) Region $\mathrm{d}<1<\left(\mathrm{d} \xi_{0} \frac{\mathrm{T}_{0}}{\mathrm{~T}_{0}-\mathrm{T}}\right)^{1 / 2}$.

We shall begin by a qualitative discussion, the detailed calculations being given in appendix A. A typical electron trajectory between times 0 and $t\left[\approx \hbar / k_{B}\left(T_{0}-T\right)\right]$ will involve a number $n=\frac{v_{F} t}{l}$ of collisions in the bulk material ("impurity" collisions) and a number $\left(-\frac{v_{F} t}{d}\right)$ of collisions on the wall; all the wall-to-wall segments do not contribute to $\phi$, as explained above. Thus we restrict our attention to segments linking an impurity to a wall, or to another impurity. Call $\Delta \phi$ the corresponding increment in phase. $\Delta \phi$ is at most of order $\frac{H}{\Phi_{0}} d 1$, but the directions of the electron velocity allowing for such a large $\Delta \phi$ are restricted to a fraction $\approx d / 1$ of all solid Angles. Thus $\left\langle\Delta \phi^{2}\right\rangle \sim\left(\frac{H}{\Phi_{0}} d l\right)^{2} d / 1 \sim\left(\frac{H}{\Phi_{0}}\right)^{2} d^{3} l$ and $\left\langle\phi^{2}(t)\right\rangle \sim n\left\langle\Delta \phi^{2}\right\rangle \sim v_{F} t\left(\frac{H}{\Phi_{0}}\right)^{2} d^{3}$. If the distribution of $\phi$ is gaussian we can apply equation (IV.1) and write

$$
\begin{aligned}
<e^{i \phi}> & =e^{-1 / 2<\phi^{2}>}=e^{-t / \tau_{K}} . \\
1 / \tau_{K} & \cong v_{F}\left(\frac{H}{\Phi_{0}}\right)^{2} d^{3}
\end{aligned}
$$

A more detailed calculation (Appendix B) gives

$$
1 / \tau_{K}=\frac{\pi^{2}}{16} v_{F}\left(\frac{H}{\Phi_{0}}\right)^{2} d^{3} .
$$


We conclude that such films belong to case II and furthermore that $\tau_{K}$ is independent of 1 . This remarkable property is very favorable (since it would be hard to measure 1 in the normal state when $l$ is larger than $d$ ). It is very important to realise that the simple result (IV.14) applies only when some rather stringent conditions are satisfied

1) $l$ is larger than $d$

2) $\phi$ has a gaussian distribution. We may discuss the latter point qualitatively by comparing $\left\langle\phi^{4}\right\rangle$ to $\left\langle\phi^{2}\right\rangle^{2}$. If $\phi=\sum_{i} \Delta \phi_{i}$ and the various $\Delta \phi$ 's are very nearly uncorrelated, we have:

$$
\begin{aligned}
\left\langle\phi^{4}\right\rangle & =3 \sum_{i \neq j}\left\langle\Delta \phi_{i}{ }^{2}\right\rangle\left\langle\Delta \phi_{j}{ }^{2}\right\rangle+\sum_{i}\left\langle\Delta \phi_{i}{ }^{4}\right\rangle \\
& \left.=3 n(n-1)\left(\left\langle\Delta \phi^{2}\right\rangle\right)^{2} n<\Delta \phi^{4}\right\rangle
\end{aligned}
$$

When the $\left\langle\Delta \phi^{4}\right\rangle$ term is negligible in (IV.15) we may write (for $n \gg 1$ )

$$
\left.\left.<\phi^{4}\right\rangle \cong 3\left(n<\Delta \phi^{2}>\right)^{2}=3<\phi^{2}\right\rangle^{2}
$$

the relation expected for a gaussian distribution. Thus we require

$$
\frac{\left.<\Delta \phi^{4}\right\rangle}{\left.n<\Delta \phi^{2}\right\rangle^{2}} \ll 1
$$

Arguing as above we estimate

$$
\left\langle\Delta \phi^{4}\right\rangle \cong\left(\frac{H}{\Phi_{0}} d l\right)^{4} \frac{d}{l} .
$$

The values of interest for $n=\frac{v_{F} t}{l}$ are $\sim \frac{h v_{F}}{k_{B}\left(T_{0}-T\right) l}$. Thus the conditions of validity of (IV.14) are

$$
d^{2}<1^{2}<d \xi_{0} \frac{T_{0}}{T_{0}-T}
$$

Making use of (IV.14) and (III.9) the second inequality may also be written

$$
H<\frac{\Phi_{0}}{d l} .
$$

b) Region d $\xi_{0} \frac{\mathrm{T}_{0}}{\mathrm{~T}_{0}-\mathrm{T}_{\mathrm{c}}}{ }^{1 / 2}<1<\xi_{0} \frac{\mathrm{T}_{0}}{\mathrm{~T}_{0}-\mathrm{T}_{\mathrm{c}}}$.

Here the moment calculation is not appropriate and it is preferable to compute $\left\langle e^{i} \quad \phi_{>}\right.$directly. Furthermore, the only segments of importance are from an impurity to a wall and vice versa (the impurityimpurity segments become negligible). Then $\left\langle e^{i} \phi_{\rangle}\right.$is simply equal to $\eta^{1 / 2}$ (the factor calculated in equation (IV.10). Each impurity collision contributes two segments, and we have

$$
\begin{gathered}
\left\langle e^{i \phi_{>}}=\eta^{n}\right. \\
\frac{1}{\tau_{K}}=\frac{v_{F}}{1} \ln \left(\frac{1}{\eta}\right)=\frac{1}{\tau} \ln \left(\frac{1}{\eta}\right)
\end{gathered}
$$


However the range of values of 1 where (IV.19) applies is severely limited by the following considerations

1) The exponential decay (III.2) is obtained only when $n=\frac{v_{F} t}{1} \gg 1$. (When $v_{F} t<1$ we have no collisions in the time intervals $t$ of interest and we return to case $I_{0}$ ) Since $t \sim \tau_{K}$ the resulting condition may be written in terms of (IV.19) as

$$
\ln \left(\frac{1}{\eta}\right)<<1
$$

or according to (IV.11)

$$
H<\frac{\Phi_{0}}{d^{2}}
$$

Another way of expressing this inequality is to use the order of magnitude relation (III.9) between $\tau_{K}$ and the transition temperature $T_{c}(H)$. This yields

$$
1<\xi_{0} \frac{T_{0}}{T_{0}-T_{0}}
$$

2) On the other hand, the major contributions to $\eta^{1 / 2}$ (equation IV.10) come from trajectories from impurity to surface of length $\sim \frac{\Phi_{0}}{H d}$. This must be smaller than 1

$$
H>\frac{\Phi_{0}}{d l}
$$

We can eliminate $H$ in terms of $\tau_{\mathrm{K}}$ by (IV.19). (Note that since (IV.20.a) must apply we may use (IV.11.a) for $\eta(H)$, and express $\tau_{K}$ in terms of $T_{c}$ by (III.9) obtaining

$$
l^{2} \gg \xi_{0} d \frac{T_{0}}{T_{0}-T_{\mathrm{c}}}
$$

4) Summary of results for thin films obeying case II. We shall now recapitulate our results for thin films, assuming that the bulk mean free path 1 is smaller than $\xi_{0} \frac{T_{0}}{T_{0}-T}$. This, as we have seen, ensures that the films belong to case II. For simplicity we restrict our attention to the critical field $H_{c}$ in the limit $\underline{T}=0 . \quad H_{c}$ is defined by the condition (III.8)

$$
\left(\frac{\hbar}{\tau_{K}}\right)_{H_{c}}=1.76 k_{B} T_{0}=\Delta_{\infty}
$$

where $\Delta_{\infty}$ is the energy gap of the bulk material at $T=0, H=0$. The coherence length $\xi_{0}$ is related to $\Delta_{\infty}$ by $\xi_{0}=\frac{\hbar v_{F}}{\pi \Delta_{\infty}}$. The explicit form for $\tau_{K}$ depends on the value of 1 .

When $\frac{d^{2}}{\xi_{0}}<1<d$ (The Maki case) $\tau_{K}$ is given by equations (IV.4) and (IV.5)

$$
H_{c}=3 \pi^{-3 / 2} \frac{\Phi_{0}}{\left(\xi_{0} l\right)^{1 / 2} d}
$$

It is sometimes convenient to rewrite the values of $H$ in terms of the bulk critical field at $T=0$,

$$
H_{\mathrm{c} b}=\sqrt{\frac{3}{2}} \frac{1}{\pi^{2}} \frac{\Phi_{0}}{\xi_{0} \lambda_{L}(0)} \text { (where } \lambda_{L}(0) \text { is the London penetration depth at } T=0 \text { ) Here we have }
$$




$$
\frac{H_{c}}{H_{c b}}=4.35 \frac{\lambda_{L}(0)}{d}\left(\frac{\xi_{0}}{1}\right)^{1 / 2}
$$

which differs from the elementary result (I.7) by only a factor of $\frac{\sqrt{\pi}}{2}=.89$

When $d<1<\sqrt{\xi_{0} 6 \text {. }}$ (case (a) of preceding section) equation (IV.14) applies and

$$
\begin{gathered}
H_{c}=4 \pi^{-3 / 2} \frac{\Phi_{0}}{\xi_{0}^{1 / 2} d^{3 / 2}} \\
\frac{H_{c}}{H_{c b}}=5.8 \frac{\xi_{0}^{1 / 2} \lambda_{L}(0)}{d^{3 / 2}}
\end{gathered}
$$

Note that in this region $H_{c}$ is independent of 1 . Again this result agrees with the simple estimate (I.8) to within a factor of $\frac{\sqrt{\pi}}{2}$.

When $\sqrt{\xi_{0} d}<1<\xi_{0}$. equations (IV.19) and (IV.11.a) apply:

$$
\begin{aligned}
& H_{c}=\frac{3}{\pi^{2}} \frac{1}{\xi_{0}} \frac{\Phi_{0}}{d^{2}} \\
& \frac{H_{c}}{H_{c b}}=2.44 \frac{1 \lambda_{L}(0)}{d^{2}}
\end{aligned}
$$

It must be emphasized, however, that (IV.26) is expected to apply only if the thickness of the film $d$ is constant to a rather good approximation. In practice, with films of thickness $\sim 500 \AA$ there will in general be some non-negligible random variations in $d$ from point to point : then the cancellation theorem (IV.8) becomes invalid and $\tau_{K}$ is modified. Qualitatively, the effect will be similar to a reduction of 1 . Thus it

may well be that in the present state of the art the regime (IV.26) is unobservable.
Finally, we briefly mention for completeness the case of very short mean free paths $1 \ll \frac{d^{2}}{\xi_{0}}$ (or $\xi(0) \ll d$ ). When $\xi<d$ we know both from theory [24] and experiment [25] that the critical field $H_{c 3}$ corresponds to the nucleation of a superconducting sheath of thickness $\xi$ on both sides of the film, and we have

$$
\frac{H_{\mathrm{c} 3}}{H_{c b}}=2.4 \kappa \approx 1.7 \frac{\lambda_{L(0)}}{l}
$$

(where $\kappa$ is the Landau Ginsburg parameter).

These results are summarized in Fig. 4.

5 ) - Spherical grains. We consider a spherical particle of radius $R$, with no defects in the bulk, but with diffuse scattering on the surface. This situation may be of interest for colloids or even for thin films of very uneven thickness which may be approximated by a two-dimensional array of such grains. Here there is a non-zero, random increment in phase between successive collisions on the surface and the magnetic behavior corresponds to case II. We shall now compute the corresponding $\tau_{K}$.

The calculation proceeds as follows: consider a segment of trajectory from point $R_{0}$ on the grain surface to point $R_{1}$, also on the surface (Fig. 5). Let $\mathbf{n}_{D}$ be a unit vector normal to the diametral plane $O R_{1} R_{2}$, and $\mathbf{n}_{z}$ a unit vector along $\mathbf{H}$. The gauge in which $\Delta$ is real is $\mathbf{A}=\frac{1}{2} \mathbf{r} \times \mathbf{H}$ where $\mathbf{r}$ is counted from the center 0 of the grain. The increment in phase $\Delta \phi$ corresponding to the path $\mathbf{R}_{0} \mathbf{R}_{1}$ is

$$
\Delta \phi=2 \pi \frac{H}{\Phi_{0}} R^{2} \sin \psi \cos \psi\left(\mathbf{n}_{D} \cdot \mathbf{n}_{z}\right)
$$




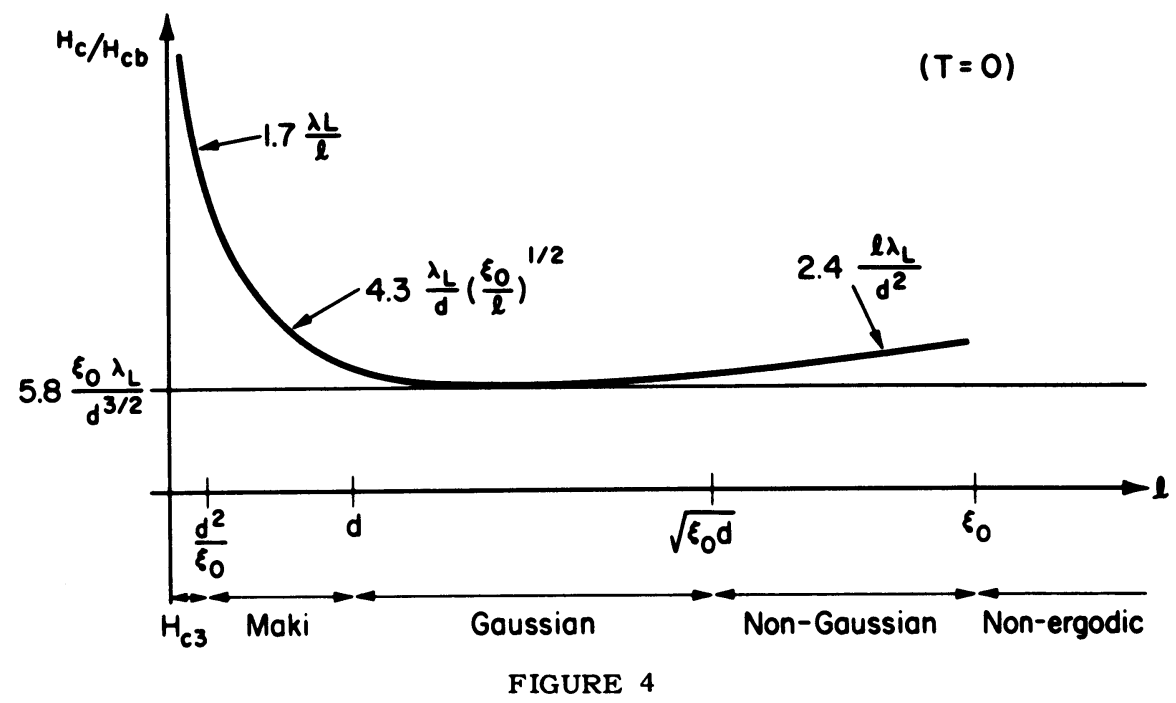

Theoretical critical field of a film of thickness $d$, as a function of the bulk mean free path 1 ( $H$ parallel to the field plane, diffuse reflection on the film boundaries). $d$ is assumed to be much smaller than the BCS coherence length $\xi_{0}$. The different regimes are discussed in section IV.

where $\psi$ is the angle between $R_{0} O$ and $R_{0} R_{1}$. The average square is

$$
\left.<\Delta \phi^{2}\right\rangle=\left(2 \pi h R^{2}\right)^{2} \int_{0}^{\pi / 2} d \psi \sin \psi \sin ^{2} \psi \cos ^{2} \psi\left(\mathbf{n}_{D} \cdot \mathbf{n}_{z}\right)^{2}
$$

Here $\left\langle\left(\mathbf{n}_{D} \cdot \mathbf{n}_{\mathbf{z}}\right)^{2}\right\rangle=1 / 3$ and

$$
<\Delta \phi^{2}>=\frac{2}{45}\left(2 \pi \frac{H R^{2}}{\Phi_{0}}\right)^{2}
$$

The average transit time is

$$
<\Delta t>=\int_{0}^{\pi / 2} \sin \psi d \psi \frac{2 R \cos \psi}{v_{F}}=R / v_{F}
$$

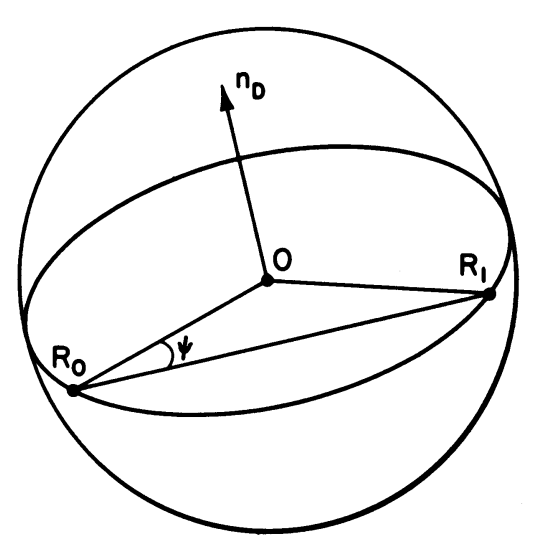

FIGURE 5

Portion of a trajectory for one electron in a spherical sample between two collisions with the sample surface at points $R_{0}$ and $R_{1}$. 
In a time interval $t \gg R / v_{F}$, the particle undergoes $n=\frac{t}{\langle\Delta t\rangle}=\frac{v_{F} t}{R}$ collisions and the average square of $\phi$ is given by

$$
\left.\left\langle\phi^{2}(t)\right\rangle=n\left\langle\Delta \phi^{2}\right\rangle=\frac{v_{F} t}{R}<\Delta \phi^{2}\right\rangle \text {. }
$$

For $n \gg 1$ we may assume that the distribution of $\langle\phi(t)\rangle$ is gaussian, and write

$$
\left\langle\mathrm{e}^{i \phi(t)}\right\rangle=\mathrm{e}^{\left.-1 / 2<\phi^{2}(t)\right\rangle}=\mathrm{e}^{-t / \tau_{K}}
$$

with

$$
\frac{1}{\tau_{K}}=\frac{2 \pi^{2}}{45}\left(\frac{H}{\Phi_{0}}\right)^{2} v_{F} R^{3} .
$$

From this result we deduce the critical field at $T=0$

$$
\begin{gathered}
H_{c}=\frac{3}{2 \pi} \sqrt{\frac{5}{\pi}} \frac{\Phi_{0}}{\xi_{0}^{1 / 2} R^{3 / 2}}=0.60 \frac{\Phi_{0}}{\xi_{0}^{1 / 2} R^{3 / 2}} . \\
\frac{H_{c}}{H_{c b}}=4.85 \frac{\lambda_{L} \xi_{0}^{1 / 2}}{R^{3 / 2}} .
\end{gathered}
$$

To relate this to our film results, let us define a "mass thickness" $d_{m}$ of an equivalent film with the same density per unit area as a close packed array of spheres of radius $R$. Then $d_{m}=\frac{2 \pi}{3 \sqrt{3}} R=1.207 R$.

Substituting in (IV.35) we obtain:

$$
\frac{H_{c}}{H_{c b}}=6.45 \frac{\lambda_{L} \xi_{0}^{1 / 2}}{d_{m}^{3 / 2}}
$$

which differs by only about $10 \%$ from (IV.25). Thus, even without volume scattering (IV.25) should be reasonably accurate for films of very irregular structure, which can be approximated by a close packed array of spheres. Since in fact very thin films are known to often have such a structure, this result considerably extends the applicability of our discussion to real films.

\section{Conclusions}

The method of one-electron trajectories presented here provides a simple but rigorous solution to the problem of determining the critical field of thin films or small spheres at all temperatures without resorting to such approximations as the Ginsburg - Landau theory. Our results show that provided $1<\xi_{0}\left(T_{0} / T_{0}-T\right)$ the critical field is finite even at $T=0$. Specific numerical results for plane films at $T=0$ are derived for the cases $1<d$ (IV.23), $d<1<\sqrt{\xi_{0}} d$ (IV.25), $\sqrt{\xi_{0}} d<1<\xi_{0}$ (IV.27). These results for the two cases with $1<\sqrt{\xi_{0}} d$ are in close agreement with the results obtained by simple but non-rigorous methods, which are known to be in quite good agreement with experiment. The case $\sqrt{\xi_{0}} d<1<\xi_{0}$ gives the novel result that $H_{c}$ increases with increasing 1 . Although there seems no experimental data to support this result, it does lead continuously to $H_{c}(0) \longrightarrow \infty$ as $l \longrightarrow \infty$ to agree with another feature of the analysis. If the film does not have completely plane parallel surfaces, these latter results come into question. Qualitatively, any roughness of the surface will prevent the approach of $H_{c}(0)$ to $\infty$, since it will prevent the geometrical cancellation of phase increments along the trajectory. It is difficult to treat this effect for an arbitrary surface, but if one approximates a very irregular film by a close-packed array of spheres, one finds that $H_{\mathrm{c}}(0)$ is essentially independent of 1 , having a value in agreement with elementary considerations. 
In all the present work our attention has been restricted to the case $\Delta \longrightarrow 0$, i.e., to the transition curve $T_{\mathrm{c}}(H)$. However, when $\phi$ has an ergodic behavior, our analysis may be extended to lower temperatures, at least to the region of the $(H T)$ plane where $\Delta$ is non-vanishing, but small (more precisely we require $\Delta \leqslant \hbar / \tau_{K}$ ). In particular, the density of states for the excitations in the superconducting state can be obtained explicitly, and it is found that when $\Delta \leqslant \hbar / \tau_{K}$ there is no energy gap [16]. From an experimental point of view, we emphasize that all Knight shift experiments and also many measurements of the nuclear relaxation time $T_{1}$ in pure superconductors have been performed on very small samples which we expect to be ergodic and intrinsically gapless in a certain range of fields. $H_{\vec{B}}<H<H_{c}$. However from a study of the Maki limit [16], we expect that $\frac{H_{c}-H_{g}}{H_{c}} \sim 5 \%$. Thus to distinguish the effects of gapless superconductivity from spurious ones due to a distribution of $H_{c}$ values the sample thickness $d$ should be controlled with an accuracy significantly better than $5 \%$.

\section{APPENDIX \\ Calculation of $\left\langle\phi^{2}\right\rangle$ for a film with I larger than $d$}

Let us first consider the increment of $\phi, \Delta \phi_{I S}$, obtained when an electron goes from an impurity site $A$ to an arbitrary point $B$ on the film surface. This is given by

$$
\Delta \phi_{I S}=\pi \frac{H}{\Phi_{0}}\left(a^{2}-x^{2}\right) \tan \psi
$$

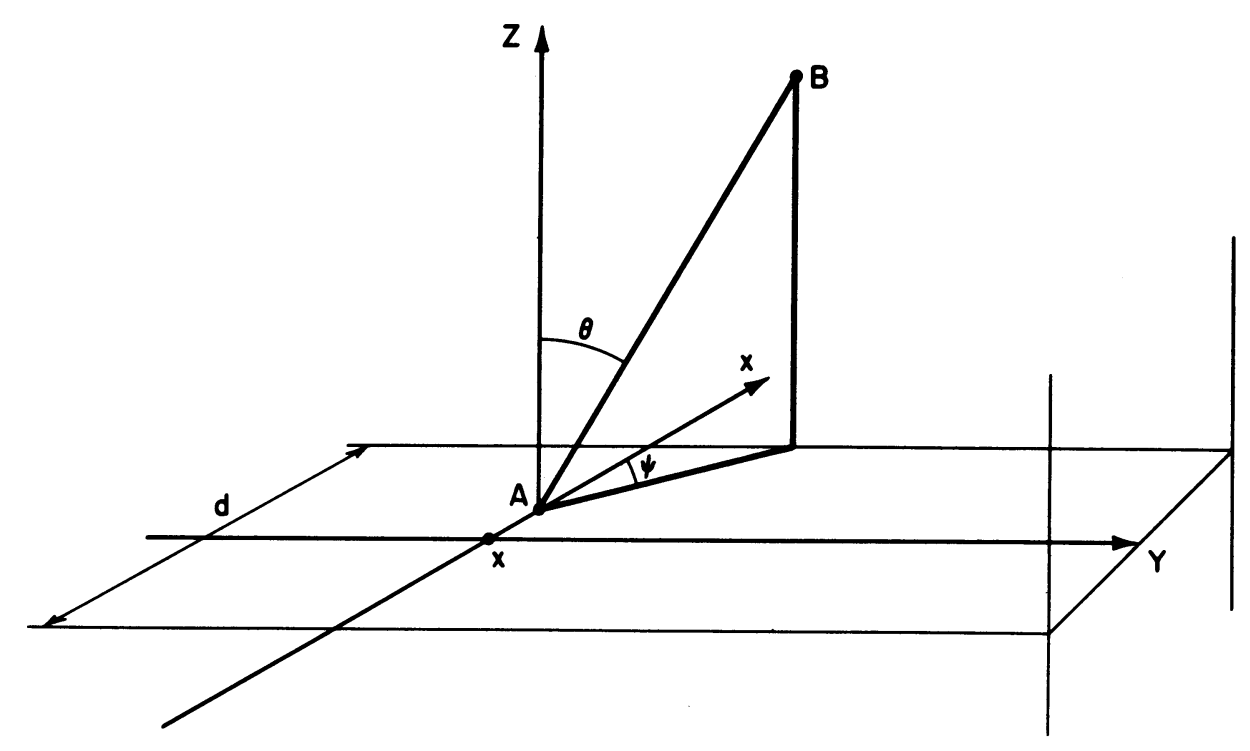

FIGURE 6

Typical trajectory from an initial point $A$ to a surface point $B$.

where $\psi$ and $x$ are defined in Fig. 6. The average square of this phase increment is given by

$$
\left\langle\Delta \phi_{I S}{ }^{2}\right\rangle=\left.\int_{-d / 2}^{d / 2} \frac{d x}{d} \int_{0}^{\pi} \frac{1}{2} \sin \theta d \theta \int_{0}^{\pi / 2} \frac{2}{\pi} d \psi \mathrm{e}^{\frac{-|d / 2-x|}{1 \cos \psi \sin \theta} \mid \frac{\pi H}{\Phi_{0}}}\left(\frac{d^{2}}{4}-x^{2}\right) \tan \psi\right|^{2}
$$

We consider the limit $1 . \gg d$. Then the important contributions in (B.2) come from $\psi$ close to $\pi / 2$, the integral can be simplified and yields

$$
\left.<\Delta \phi_{I S}{ }^{2}\right\rangle=\frac{1}{3}\left(\frac{\pi H}{\Phi_{0}}\right)^{2} 1\left(\frac{d}{2}\right)^{3}
$$


We also have to consider the increment $\Delta \phi_{S I}$ corresponding to a trajectory from the surface to an impurity: clearly $\left\langle\Delta \phi_{S I}{ }^{2}\right\rangle=\left\langle\Delta \phi_{I S}{ }^{2}\right\rangle$. Finally we need to consider the increment $\Delta \phi_{I I}$ corresponding to a segment of trajectory relating two impurities. If $R$ is the distance between impurities, $\theta$ and $\psi$ the polar angles of the trajectory, and $x$ the abscissa of the first impurity, we have:

$$
\begin{gathered}
\Delta \phi_{I I}=2 \pi \frac{H}{\Phi_{0}} R \sin \theta \sin \psi\left(x+\frac{1}{2} R \sin \theta \cos \psi\right) . \\
\left\langle\Delta \phi_{I I}^{2}\right\rangle=\int_{-d / 2}^{d / 2} \frac{d x}{d} \int_{0}^{\pi} \frac{\sin \theta d \theta}{2} \int_{0}^{\infty} e^{-R / 1} \frac{d R}{1} \int_{-\pi / 2}^{\pi / 2} \frac{d \psi}{\pi} \Delta \phi_{I I}^{2} .
\end{gathered}
$$

Again in the limit $l \gg d$ the integral can be simplified and gives

$$
\left\langle\Delta \phi_{I I}^{2}\right\rangle=\frac{1}{3}\left(\frac{\pi H}{\Phi_{0}}\right)^{2} 1\left(\frac{d}{2}\right)^{3}=\left\langle\Delta \phi_{I S}^{2}\right\rangle=\left\langle\Delta \phi_{S I}^{2}\right\rangle \text {. }
$$

Finally if we consider a complete trajectory between times 0 and $t$ we can write

$$
\left\langle\phi^{2}\right\rangle=\left\langle\Sigma\left(\Delta \phi_{I S}^{2}+\Delta \phi_{S I}^{2}+\Delta \phi_{I I}^{2}\right)\right\rangle
$$

since all segments from surface to surface do not contribute according to the cancellation theorem (IV.8).

The number of collisions on impurities is $n=\frac{v_{F} t}{l}$ and each impurity collision contributes once to each of three terms on the right side of (B.6). Thus

$$
\left\langle\phi^{2}\right\rangle=n 3\left\langle\Delta \phi_{I I}^{2}\right\rangle=v_{F} t\left(\frac{\pi H}{\Phi_{0}}\right)^{2}\left(\frac{d}{2}\right)^{3} .
$$

If the distribution of $\phi$ is gaussian we may then write

$$
\left\langle e^{i \phi}\right\rangle=e^{-1 / 2\left\langle\phi^{2}\right\rangle}=e^{-t / r_{K}}
$$

with

$$
\frac{1}{\tau_{K}}=\frac{\pi^{2}}{16} v_{F}\left(\frac{H}{\Phi_{0}}\right)^{2} d^{3}
$$

\section{References}

1. See for example, D. SHOENBERG, Superconductivity. Cambridge University Press (1952).

2. V. L. GINSBURG and L. D. LANDAU, Zh. Eksp. i Teor. Fiz. 20, 1064 (1950).

3. D. H. DOUGlaSS, JR., Phys. Rev. Letters 6, 346 (1961).

4. A. B. PIPPARD, Proc. Roy. Soc., A 216, 547 (1953).

5. A. A. ABRIKOSOV, Dok. Akad. Nauk SSSR 86, 489 (1952); Zh. Eksp. i Teor. Fiz. 32, 1442 (1957) [translation: Soviet Physics, J.E.T.P. 5, 1174 (1957)].

6. P. G. DE GENNES, Cours sur les Métaux et Alliages Supraconducteurs, Orsay 1963; also Rev. Mod. Phys. 36, 312 (1964).

7. J. BARDEEN, L. N. COOPER and J. R. SCHRIEFFER, Phys. Rev. 108, 1115 (1957).

8. P. B. MILlER, Phys. Rev. 113, 1209 (1959).

9. K. T. ROGERS, Ph.D. Thesis, University of Illinois (1960) unpublished.

10. A. M. TOXEN, Phys. Rev. 127, 382 (1962).

11. J. BARDEen, Rev. Mod. Phys. 34; 667 (1962).

12. D. E. MORRIS and M. TINKHAM, Phys. Rev. Letters 6, 600 (1962); Phys. Rev. to be published. 
13. R. MESERVEY and D. H. DOUGLASS, Phys. Rev. to be published.

14. Y. NAMBU and S. F. TUAN, Phys. Rev. Letters 11, 119 (1963); Phys. Rev. 133, A1 (1964); Rev. Mod. Phys. 36, 288 (1964).

15. C. D I CASTRO and J. G. VALATIN, Phys. Letters 8, 230 (1964).

16. K. MAKI, Prog. Theor. Phys. 29, 603 (1963); and to be published.

17. P. G. DE GENNES and G. SARMA, J. Appl. Phys. 34, 1380 (1963).

18. P. G. DE GENNES, Rev. Mod. Phys. 36, 225 (1964).

19. L. P. GOR'KOV, Zh. Eksp. Theor. Fiz. 37, 833 (1959) [translation: Soviet Physics, J.E.T.P. 10, 593 (1960)].

20. A. ABRIKOSOV and L. P. GOR'KOV, Zh. Eksp. i Teor. Fiz. 39, 1781 (1960); [translation: Soviet Physics, J.E.T.P. 12, 1243 (1961)].

21. D. H. DOUGLASS and R. H. BLUMBERG, Phys. Rev. 127, 2038 (1962).

22. A. M. TOXEN, Rev. Mod. Phys. 36, 308 (1964).

23. Below the transition point it is sometimes of interest to consider situations where an external current is applied and to modify (IV.7.b) accordingly. Note in this connection that when $\frac{2 \mathrm{e} A}{\hbar \mathrm{c}}=q=$ constant, equation (IV.4) gives $\frac{1}{\tau_{K}}=D q^{2}$ (where $D$ is the diffusion coefficient), a relation derived directly by DE GENNES and GUYON, Phys. Letters 3, 128 (1963).

24. D. SAINT JAMES and P. G. DE GENNES, Phys. Letters 7, 306 (1963).

25. See for instance J. P. BURGER, G. DEUTSCHER, E. GUYON, and A. MARTINET, Solid State Comm. 2, 101 (1964), where other references are quoted.

26. L. P. GOR'KOV, Zh. Eksp. i Teor. Fiz. 36, 1918 (1959); [translation: Soviet Physics, J.E.T.P. 9, 1364 (1959)]. 